\title{
Effect of $E$. coli antigens, tuberculin, and phytohaemagglutinin upon ulcerative colitis lymphocytes
}

\author{
S. STEFANI AND S. FINK
}

From the Veterans Administration Hospital and Loyola Stritch School of Medicine, Hines, Illinois, U.S.A.

EDITORIAL COMMENT Some very interesting observations showing that lymphocytes from the peripheral blood in patients with active ulcerative colitis differed from other lymphocytes tested in their response to $E$. coli extracts.

Human peripheral blood lymphocytes respond in vitro to a wide range of agents by undergoing blastoid transformation and mitosis (Pearmain, Lycette, and Fitzgerald, 1963; Elves, Roath, and Israels, 1963). It is believed that specific antigenantibody reactions within the growing cells are responsible for these changes (Hashem, 1965). As a result of these observations, Hirschhorn, Back, Kolodny, Firschein, and Hashem (1963), among others, have stated that the human peripheral lymphocyte provides a readily available system representative in vitro of the donor's immune status.

It has been suggested that the destruction of colonic mucosa in ulcerative colitis is the result of an immunological reaction in which $E$. coli may play a role (Perlmann, Hammarström, Lagercrantz, and Gustafsson, 1965). It was, therefore, decided to study in vitro the morphological reaction of blood lymphocytes obtained from patients with ulcerative colitis and from normal donors to extracts of a pathogenic strain of $E$. coli $(0119:$ B 14$)$ as well as to old tuberculin and phytohaemagglutinin.

\section{METHOD}

Lymphocytes were obtained from three groups of ambulatory adult male donors. Two groups of patients had known ulcerative colitis established by barium enema and sigmoidoscopy and confirmed by compatible rectal biopsy. The first of these was judged to have active ulcerative colitis by the presence of diarrhoea, rectal bleeding, and gross proctoscopic abnormalities, graded three and four (Dragstedt, Dack, and Kirsner, 1941) at the time of the study in vitro. The patients in the second group were free of symptoms, had normal mucosal appearance on proctoscopy and were, therefore, classified as being in remission. Patients with mild or no symptoms but with mucosal abnormalities were not included in this investigation. The third group consisted of normal donors. None of the patients studied had received corticosteroid therapy during the six months preceding the investigation. As indicated in Table I, 10 patients were taking salicylazosulphapyridine (Azulfidine) and had been receiving the drug for periods of six to 24 months.

Twenty millilitres of blood were obtained from each donor and sedimentation was carried out for 90 minutes at $37^{\circ} \mathrm{C}$. The leucocytes in the supernatant plasma were centrifuged, washed, and resuspended in the final medium which consisted of equal parts of TC 199 (Difco) and a compatible human serum. The suspension was then introduced into a flat-bottomed $\mathrm{T}$ flask and incubated for 30 minutes at $37^{\circ} \mathrm{C}$. During this procedure, the granulocytes and monocytes adhere to the glass. In the supernatant, 90 to $99 \%$ of the nucleated cells are seen to be lymphocytes. The supernatant was divided into $0.9 \mathrm{ml}$. aliquots and placed in small test tubes. From each patient, one group of lymphocyte suspensions was treated with $0.1 \mathrm{ml}$. of saline and served as a control, another group with phytohaemagglutinin (PHA-P Difco) at a final concentration of $4 \mu \mathrm{l}$. per ml., another with old tuberculin (Parke-Davis \& Co.) at a final concentration of $1: 100$. The last three groups were treated with each of three extracts derived from cultures of $E$. coli 0 119: B 14. Two of the three $E$. coli extracts, a protein and a polysaccharide, were prepared by the phenol extraction method of Westphal et al., (1952); the third, a K antigen, was kindly supplied by the Sylvana Company, Millburn, New Jersey. Rabbit antisera to each of the E. coli extracts were prepared and the antigenic potency of the three preparations was verified by precipitin-ring tests. The suspensions were incubated for six days at $37^{\circ} \mathrm{C}$. On the sixth day, several slide chambers were prepared from each suspension and the viable cells were examined, photographed, and counted by use of an inverted phase contrast microscope (Schrek and Stefani, 1964). Blastoid cells were arbitrarily defined as those having $10 \mu$ as 
their smallest diameter. At least 1,000 lymphocytic cells were counted in each group of suspensions derived from any given donor and the number of blastoid cells and mitotic figures present was expressed as a percentage of this total lymphocytic cell count. The suspensions were classified according to the total percentage yield of blastoid cells and mitotic figures as follows:

$++++=>20 \%$ blastoid cells or mitotic figures $+++=10 \%$ to $20 \%$ blastoid cells or mitotic figures $++\quad=5 \%$ to $10 \%$ blastoid cells or mitotic figures $+\quad=<5 \%$ blastoid cells or mitotic figures

$0=$ no blastoid cells and mitotic figures, or only a few, but not in all slides examined

The cells were classified without foreknowledge on the part of the examiner either of the donor's diagnosis or of the agent used.

\section{RESULTS}

The results are presented in Table I. The salinetreated suspensions produced no blastoid transformation while suspensions treated with phytohaemagglutinin showed a uniform marked response regardless of the donor's diagnosis and stage of the disease. Old tuberculin produced constant blastoid transformation and formation of mitosis in all lymphocyte suspensions tested from purified protein derivative (P.P.D.) positive patients but not in suspensions from patients who were P.P.D. negative. The morphological response was irrespective of the phase of the patient's disease. This response to old tuberculin of lymphocytes from patients with ulcerative colitis was comparable to that observed many times in this laboratory in lymphocytes from normal donors (Stefani, 1966). Lymphocyte suspensions exposed to $E$. coli extracts showed a different pattern of response. Lymphocytes obtained from the control group and from patients with ulcerative colitis in remission showed consistent blastoid transformation with all the $E$. coli extracts although this reaction was usually most marked in the presence of the protein extract. In contrast, all three $E$. coli extracts induced little or no blastoid transformation in the lymphocyte suspensions obtained from patients with ulcerative colitis in an active phase.

TABLE I

REACTIVITY in vitro OF NORMAL AND ULCERATIVE COLITIS LYMPHOCYTES ${ }^{1}$

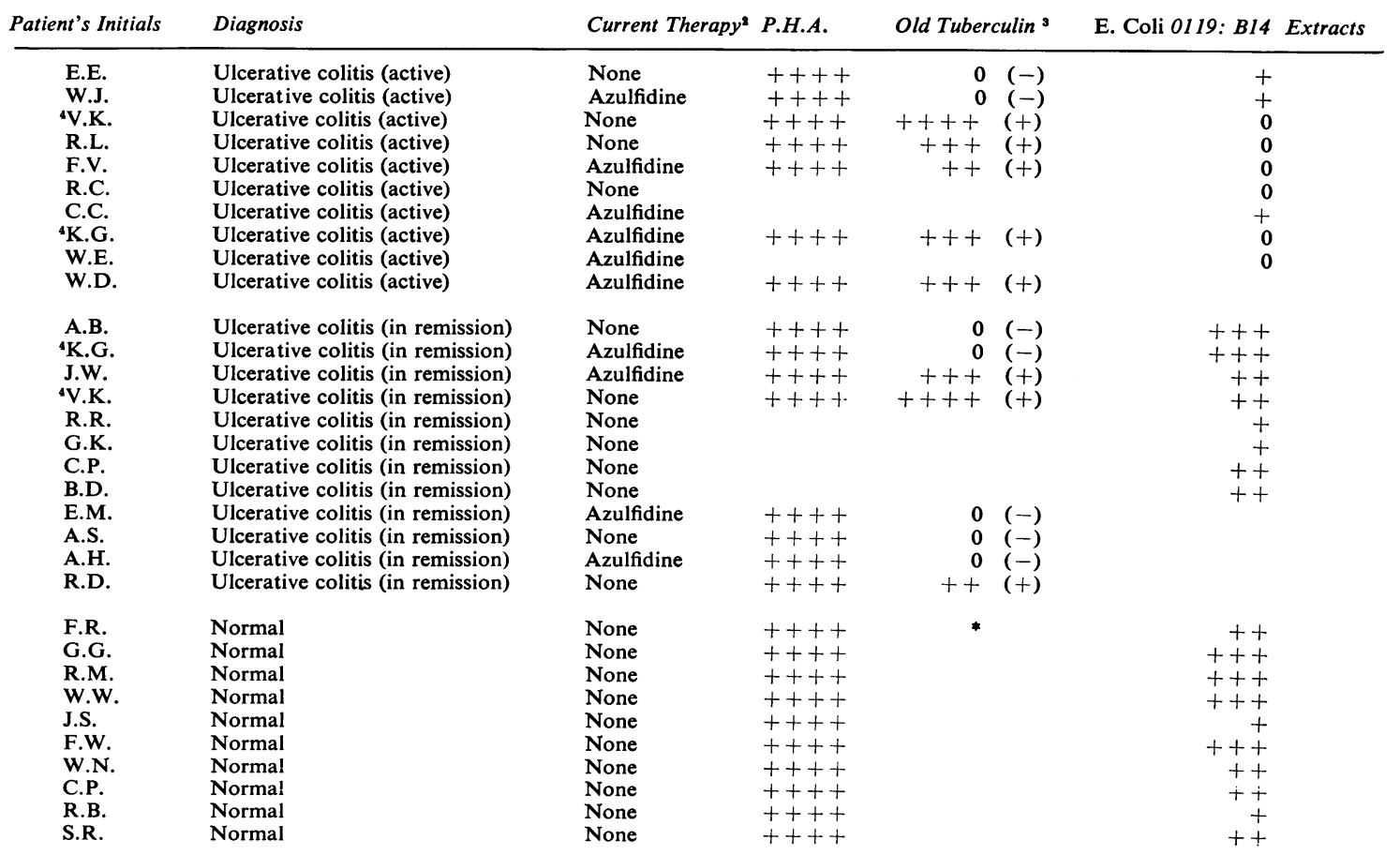

${ }^{1}$ See classification of reactivity in text.

${ }^{2}$ During the six months preceding the investigation.

${ }^{3}$ The sign in parenthesis in this column represents the status of the tuberculin test with intermediate P.P.D. strength.

"These two patients changed to a different phase of their disease during the investigation.

* All lymphocyte suspensions from normal donors studied in this laboratory by this technique gave a ++ to ++++ response if P.P.D. positive and 0 if P.P.D. negative (Stefani, 1966). 
Some patients both in remission and in the active phase of the disease were receiving Azulfidine treatment at the time of the investigation. No correlation was observed between this treatment and the degree of $E$. coli-induced blastoid transformation.

Two patients, V. K. and K.G., were examined twice at three-and five-month intervals respectively. During the first investigation, V.K. was classified as having active ulcerative colitis and no morphological response to $E$. coli extracts was detected. At the time of the second study, he was in remission and blastoid transformation $(++)$ was observed. On the other hand, K.G. was first classified as being in remission and full blastoid transformation $(+++)$ was observed. No reaction was detected when this patient was examined five months later during an exacerbation of the disease.

The possibility that a serum factor might interfere with the blastoid transformation in patients with active ulcerative colitis was investigated. Sera from two patients with active ulcerative colitis were added to lymphocyte suspensions taken from two patients in the control group, and no reduction in the extent of blastoid transformation was observed. Similarly, the addition of normal sera to suspensions of lymphocytes from patients with active ulcerative colitis failed to modify the reaction, i.e., no blastoid transformation occurred.

\section{DISCUSSION}

The phenomenon of blastoid transformation of lymphocytes in vitro in the presence of a specific antigen such as old tuberculin or of phytohaemagglutinin is well known (Pearmain et al., 1963; Elves et al., 1963). Human blood lymphocytes from all of our patients with ulcerative colitis exhibited a normal reaction to old tuberculin and phytohaemagglutinin. The present experiment revealed, however, that peripheral blood lymphocytes from patients with active ulcerative colitis differed from the other lymphocytes tested in their response to $E$. coli extracts, i.e., they did not show the expected blastoid transformation. This phenomenon varied in the same patient according to the stage of his disease and seemed to be independent of Azulfidine therapy. Moreover, this inhibitory effect was not due to a factor present in the serum of ulcerative colitis patients as shown by the experiments with media prepared with ulcerative colitis serum. Hence, the most probable explanation for this finding is that ulcerative colitis lymphocytes have at least one dysfunction demonstrable only during the acute stage of the disease when the cell is challenged with E. coli extracts.
On the basis of experimental and clinical observations, it has been suggested that ulcerative colitis is an autoimmune disease in which the lymphocyte plays a central role (Kirsner, 1965). The finding of some cross reactivity between certain $E$. coli extracts and colonic mucosal antigens (Perlmann et al., 1965 ) suggests that $E$. coli may sensitize the lymphocytes of patients with ulcerative colitis with subsequent damage to the colon by these cells (Kirsner, 1965). Our finding that ulcerative colitis lymphocytes present an impaired reaction to $E$. coli antigens would seem to weaken the hypothesis of a high lymphocyte reactivity to $E$. coli as an essential factor in the pathogenesis of the condition. Two alternative possibilities should be considered: one, that this impaired reaction may indicate a diminished defence, and thereby permit damage of the colonic mucosa by pathogenic strains of $E$. coli such as 0119 : B 14 . The second alternative is that the diminished lymphocyte response is caused by lymphocyte 'exhaustion' due to prolonged stimulation from $E$. coli which freely enters the body through the ulcerating mucosa. This impaired reaction would follow the destruction of the mucosa and would be a secondary phenomenon in the course of acute ulcerative colitis. However, it should be emphasized that only one strain of $E$. coli was used in this experiment; it is possible that the ulcerative colitis lymphocytes will respond differently to extracts obtained from other strains.

\section{SUMMARY}

Peripheral blood lymphocytes of 20 patients with ulcerative colitis and 10 normal patients were cultured in the presence of old tuberculin, phytohaemagglutinin, and extracts of $E$. coli 0 119: B 14. After six days of incubation, the degree of blastoid transformation and mitosis was determined by phase contrast microscopy. Lymphocytes from all ulcerative colitis patients studied exhibited a normal reaction to phytohaemagglutinin and old tuberculin. However, lymphocytes from patients with active ulcerative colitis differed from lymphocytes of patients with the disease in remission and from normal donors in their reaction to $E$. coli extracts. The observed absent or diminished reaction of lymphocytes from active ulcerative colitis patients was found to be independent of Azulfidine therapy and of serum factors. The degree of reactivity varied according to the change in stage of the disease for two patients in whom a second study was made.

We thank Mr. M. Carino, Mr. C. Kirsh, and Mrs, I. Zielonka for technical assistance. This work was partially supported by research grant AM 097930-3 from the National Institutes of Health, Bethesda, Maryland. 


\section{REFERENCES}

Dragstedt, L. R., Dack, G. M., and Kirsner, J. B. (1941). Chronic ulcerative colitis. A summary of evidence implicating bacterium necrophorum as an etiologic agent. Ann. Surg., 114, 653-662.

Elves, M. W., Roath, S., and Israels, M. C. G. (1963). The response of lymphocytes to antigen challenge in vitro. Lancet, 1, 806-807.

Hashem, H. (1965). Mitosis: Induction by cultures of human peripheral lymphocytes. Science, 150, 1460-1462.

Hirschhorn, K., Back, F., Kolodny, R. L., Firschein, I. L., and Hashem, H. (1963). Immune response and mitosis of human peripheral blood lymphocytes in vitro. Ibid., 142, 1185-1187.

Kirsner, J. B. (1965). The immunologic response of the colon. $J$. Amer. med. Ass., 191, 809-814.
Pearmain, G., Lycette, R. R., and Fitzgerald, P. H. (1963). Tuberculininduced mitosis in peripheral blood lymphocytes. Lancet, 1, 637-638.

Perlmann, P., Hammarström. S., Lagercrantz, R., and Gustafsson, B. E. (1965). Antigen from colon of germ-free rats and antibodies in human ulcerative colitis. Ann. N.Y. Acad. Sci., 124, 377-394.

Schrek, R., and Stefani, S. (1964). Radioresistance of phytohaemagglutinin-treated normal and leukemic lymphocytes. J. nat. Cancer Inst., 32, 507-521.

Stefani, S. (1966). Old tuberculin-induced radioresistance on human lymphocytes in vitro. Brit. J. Haemat., 12, 345-350.

Westphal, O., Luderitz, O., and Bester, F. (1952). Uber die Extraction von Baktieren mit Phenol/Wasser. Z. Naturprsch., 70, 148-155. 